\title{
APORTACIONES Y POTENCIALIDADES DE LA ANTROPOLOGÍA DE LA SALUD
}

\section{Carmen Mozo}

\section{Universidad de Sevilla}

\section{Grupo de Investigación GEISA}

La Antropología de la Salud constituye un campo de estudio plenamente consolidado y prolífico. Lejos queda ya el momento en el que Comelles (1988:551) escribía que el reto pasaba, "en el momento presente, más que por una exposición de hallazgos o de logros, por una discusión de problemas teóricos, metodológicos y conceptuales"1. En la actualidad, la Antropología de la Salud² parte de unos tópicos compartidos, de unos

1. En relación al panorama internacional, una década antes Arthur Kleinman había escrito que "La mayor parte de la literatura [antropológico-médica] está compuesta de estudios empíricos, que no suelen especificar el marco teórico que emplean, que importan asistemáticamente conceptos tanto de las ciencias sociales como de la biomedicina, y que, por tanto, son fragmentarios y difíciles de poner en relación. Poseemos un gran abanico de descripciones empíricas, pero pocas comparaciones transculturales, lo cual dificulta cualquier intento de comprobar hipótesis específicas" (Kleiman, 1978:85) (la traducción es mía).

2. La denominación más institucionalizada y generalizada para este campo de estudios es "Antropología Médica". Sin caer en discusiones nominalistas y a pesar de que, dado que "las Ciencias Antropológicas y Sociales siguen trabajando básicamente sobre la enfermedad, por lo cual yo sigo hablando de Antropología Médica, y no de Antropología de la Salud como algunos compañeros han propuesto" (Menéndez, 2012), el término “Antropología de la Salud” me parece más holístico e inclusivo. 
conceptos básicos, de una complejidad teórica y metodológica, de una comprensión de qué puede aportar la Antropología al análisis de los procesos de salud y enfermedad. No es mi intención realizar una genealogía ni un estado de la cuestión de este campo de estudios. Para ello existen ya excelentes revisiones ${ }^{3}$. Pero sí explicitar cuáles son, en mi opinión, sus potencialidades.

La primera de ellas reside en su capacidad para contribuir a desvelar el orden social. Todo proceso de salud/enfermedad/atención (Menéndez, 1994) es un proceso estructural, cuya resolución es necesaria para la producción y reproducción de cualquier sociedad. Dicha resolución no se produce en abstracto, sino inserta en el seno del sistema de desigualdades sociales que articula esa sociedad, cuya lógica incorpora. Por tanto, cuando analizamos los procesos de salud y enfermedad, no estamos estudiando fenómenos exóticos o periféricos sino, por el contrario, fenómenos que posibilitan una puerta de acceso directa a la comprensión del "núcleo duro" de la vida social.

La segunda de las potencialidades que me gustaría destacar es su posicionamiento, tremendamente crítico, con la teoría y la práctica biomédica, con lo que Menéndez (1978) denominó Modelo Médico Hegemónico, fundamentalmente con la reducción biologicista de su mirada. La biomedicina ${ }^{4}$, como cualquier sistema médico, no es sino un sistema sociocultural más, con su grado, mayor o menor, de eficacia. Mayor, qué duda cabe, en los procesos agudos; menor, qué duda cabe también, en los procesos leves y en los crónicos... 5 . Y dado que, como se infiere de articular esta segunda con la primera de las potencialidades señaladas, la biomedicina responde a la lógica social de las sociedades de mercado en las que se originó y consolidó, estudiar críticamente la teoría y la práctica biomédica va a desvelar, a la vez que cuestionar, el profundo sistema de desigualdades que articula nuestra sociedad. Efectivamente, la forma que tiene nuestra sociedad, a través de sus políticas e instituciones sanitarias de resolver (o de no resolver), de afrontar (o de no afrontar) los problemas de salud de la ciudadanía (o de quienes no son ciudadanos); incluso la definición de lo que se considera (y de lo que no se considera) un problema de salud o de lo que se califica (o no) como enfermedad; es un reflejo claro de las relaciones socioeconómicas, de sexo-género, sexuales y étnicas que la vertebran.

3. Para el caso del Estado español, por citar algunas de las más significativas, pueden consultarse Comelles y Martínez (1993) o Martínez (1996).

4. Medicina científica, medicina alopática, medicina occidental son otras de sus denominaciones. Biomedicina me parece el término que mejor la define ya que es el biologicismo su rasgo clave, estructural.

5. Cuando en los años ochenta del siglo XX autores como Kleinman (1980) asientan la idea de que el sistema biomédico constituye una etnomedicina más, un conjunto de creencias y prácticas, entre otras posibles, que un colectivo humano elabora sobre los procesos de salud y enfermedad, se abre un amplio abanico de posibilidades para la Antropología de la Salud. 
Un caso paradigmático que puede servirnos para ilustrar estas afirmaciones es el de la fibromialgia ${ }^{6}$. A la luz de las premisas anteriores, la fibromialgia ya no es solo, ni necesariamente, una "mera" patología que ha de ocupar a clínicos, terapeutas "alternativos", asociaciones de mujeres afectadas y a instituciones sanitarias, públicas o privadas. ¿Qué es lo que nos interesa de esta nueva enfermedad? Entre otras cosas, y fundamentalmente, el hecho de que, hasta el momento, afecta mayoritariamente a mujeres ${ }^{7}$, presentando un pico de prevalencia situado entre los 40 y 49 años. Además, mujeres de niveles socioeconómicos medio-bajos, con conflictos familiares, a menudo con presencia de hechos traumáticos en sus trayectorias vitales (no es un dato menor la aparición de episodios de abusos sexuales), polimedicadas, muchas ya diagnosticadas con depresión. Datos todos ellos que, para ser coherentes con lo planteado como aportaciones y potencialidades analíticas de la Antropología de la Salud, nos conminan a prestar atención a cómo las relaciones sociales de sexo (aunque no solo ellas) vertebran la enfermedad.

En nuestra sociedad, convendremos, y creo que esto es un punto obligado de partida, que dichas relaciones son relaciones estructurales vehiculadas por el poder en la medida en que ocupar una posición de sexo u otra, con independencia de nuestra voluntad, provoca que tengamos, como plantea Joan Scott (1990), un acceso diferencial, y desigual, a los recursos tanto materiales como simbólicos de nuestra sociedad. Si, por tanto, no podemos pensar la salud de las mujeres -ni la de los hombres- al margen de su posición social en cuanto mujeres - $\mathrm{u}$ hombres- de su sociedad, habría que reflexionar sobre cuál es hoy por hoy la situación social de las mujeres y no patologizar, y así individualizar, lo que en muchos casos podrían ser síntomas y expresiones de malestares sociales. Habría así que profundizar en la hipótesis de que la fibromialgia pueda ser entendida como una palabra para etiquetar un dolor y un malestar profundo y generalizado que se manifiesta

6. Según el Ministerio de Sanidad, "La Fibromialgia (FM) es una afección crónica de etiología desconocida, caracterizada por la presencia de dolor crónico musculoesquelético generalizado, que suele coexistir con otros síntomas, fundamentalmente fatiga y problemas de sueño, pero también pueden estar presentes parestesias, rigidez articular, cefaleas, sensación de tumefacción en manos, ansiedad y depresión, problemas de concentración y memoria" (2011:29). Fue reconocida por la OMS en 1992 y clasificada en el manual de Clasificación Internacional de Enfermedades (CIE-10) en su última revisión con el código M79.0. La subjetividad de su sintomatología (dolor generalizado, falta de sueño, cansancio, malestar emocional...) dificulta el diagnóstico a un sistema médico que requiere datos objetivos para el mismo.

7. Según una de las fuentes más citadas tanto por instancias oficiales como académicas, el "Estudio sobre prevalencia de enfermedades reumáticas en la población española (EPISER)”, realizado en el año 2000 por la Sociedad Española de Reumatología, afecta al 2,4\% de la población española, con una clara prevalencia en mujeres: el $4,2 \%$ frente al $0,2 \%$ en hombres. 
en el cuerpo, proveniente de las discriminaciones que sufren las mujeres en nuestra sociedad (y trabajar a su vez con su articulación con la clase social). Esta hipótesis vendría agravada, además, por una teoría y una práctica científica androcéntrica y sexista. Y es en este sentido que autoras como la médica Carme Valls-Llobet (2009) señalan cómo el diagnóstico diferencial es todavía una "asignatura pendiente", apuntando hacia la existencia de muchas patologías y disfunciones, con mayor prevalencia en mujeres, que están en la base biológica de un dolor que se cronifica porque no se trata adecuadamente. Ante lo cual la pregunta sería: ¿por qué no se diagnostica un dolor que en la mayoría de los casos tiene tratamiento "antes de decidir que una persona tiene una fibromialgia de origen desconocido y que no tiene más tratamiento que sicofármacos, analgésicos, opiáceos o derivados de la morfina?" (2009:188). Hablar de maltrato médico, tal y como hace la autora, no parece, en este contexto, descabellado.

Reteniendo y retomando las potencialidades señaladas, podemos afirmar que los textos agrupados en este monográfico, por lo demás claramente misceláneo por los temas que aborda, por los enfoques teóricos que incorpora y por el tipo de textos que incluye, contribuyen, de una u otra manera, a los propósitos expuestos: crítica al acto curativo biomédico y al proceso de medicalización de la vida (Marcial Gondar), denuncia de la función de la biomedicina como reguladora del orden socio-sexual (Fernando Tena), explicitación de las relaciones entre práctica biomédica y desigualdad social (Paola Sesia), articulación entre tecnología médica y concepciones culturales (Gemma Orobigt, Joan Bestard y Carles Salazar). En las páginas que siguen singularizaré las aportaciones de cada uno de ellos en relación a las potencialidades subrayadas.

El texto de Marcial Gondar, "El factor cultural como herramienta terapéutica. A vueltas con el modelo biomédico en la atención sanitaria", se preocupa por contribuir a mejorar la eficacia de la atención sanitaria biomédica poniendo de relieve la importancia de tener en cuenta la dimensión cultural de la enfermedad y, más en concreto, de utilizar el factor cultural y lingüístico de un pueblo como herramienta de curación, tomando como referente empírico la cultura campesina gallega. La novedad de su texto, sin embargo, no reside en señalar esta cuestión. Su artículo no es una reflexión teórica que advierte sobre la importancia de la dimensión cultural de la enfermedad, cuestión suficientemente señalada en la literatura antropológica, sino que se sitúa en un marco aplicado en el que propone "estrategias sacadas del Marketing y de la Pragmática" para aplicar en la relación terapéutica, argumentando la eficacia de las mismas.

En tanto que preocupado por mejorar la eficacia terapéutica biomédica, Marcial Gondar se sitúa en la línea de la llamada "Antropología de la illness" que insiste en la necesidad de recuperar la perspectiva del paciente, su cosmovisión, porque de esta manera se está teniendo en cuenta un elemento fundamental para la curación: la elaboración significativa de su experiencia. La importancia de tener en cuenta las cosmovisiones de los pacientes así como la importancia dada a la comunicación, entendida como 
una retroalimentación, entre médico y paciente, supone re-considerar a estos últimos y situarlos como sujetos sociales y no como meros objetos de intervención médica. Además, su texto no se focaliza únicamente en la relación médico-paciente, tal y como se ha criticado a este enfoque, sino que incorpora la importancia del contexto social. La creencia en la centralidad de las relaciones sociales y del sentido de pertenencia a una "comunidad" para una vida saludable lleva al autor a proponer (y ejemplificar) un tipo de intervención sobre la realidad en la que los medicamentos no lo son todo: recetar sociabilidad, promoverla a través de actuaciones prácticas y concretas, es hacer Medicina Preventiva, es hacer "medicina desmedicalizada".

Retomando la importancia del contexto social, el articulo de Fernando Tena, "Sacudirse la tutela médica. Hacia la despatologización de la transexualidad" nos conduce hacia un tipo de análisis más situado en una "Antropología de la Sikness", atenta al tipo de relaciones sociales estructurales a través de las que se vehiculan los procesos de salud y enfermedad, que incorpora además y bebe de los análisis feministas, con quienes el autor comparte esa voluntad política de contribuir a transformar el orden social.

Estudiar la transexualidad se convierte en un leiv-motiv para reflexionar sobre cómo la biomedicina actúa como un instrumento regulador y controlador de las transgresiones, en este caso de las transgresiones al sistema sexo-género en nuestra sociedad. Es claro que no solo el modelo biomédico cumple funciones de control, de normativización y de legitimación. Todos los sistemas médicos lo cumplen. Pero, como señalábamos, la potencialidad de la Antropología de la Salud reside precisamente en explicarnos la lógica y el funcionamiento de uno de los instrumentos más potentes a través de las cuales se ejercen estas funciones en nuestra sociedad.

Analizar el papel de la biomedicina como reguladora de las transgresiones al género conduce también a denunciar el orden social, más concretamente el socio-sexual. En este caso, el autor ha elegido centrarse en cómo son ya los propios sujetos sociales definidos como patológicos los que han asumido ese papel de denuncia a través de la Red por la Despatologización Trans del Estado español, articulada a nivel internacional, que está pidiendo la revisión del DSM y de la $\mathrm{CIE}^{8}$ para que la transexualidad deje de ser considerada una enfermedad.

Si la medicalización es un mecanismo para individualizar y dejar intacto el orden social, patologizando en este caso a las personas cuya vivencia identitaria diverge de la definida como normal (esto es, a un sexo fenotípico definido en base a la genitalidad, la identificación con un género social) y proponiéndoles una adecuación del sexo al género sentido; lo que una parte del movimiento transexual está empezando a hacer es invertir

8. La siglas DSM significan Diagnostic and Statistical Manual of Mental Disorders, de la Asociación Americana de Psiquiatría; las siglas CIE, Clasificación Internacional de Enfermedades de la Organización Mundial de la Salud. 
la ecuación: es la sociedad la que crea la enfermedad, definiendo como patológicos a quienes no encajan en la rigidez de un sistema sexo-género dicotómico y biologicista y proponiéndoles un itinerario terapéutico que tiene como objetivo adecuarles al orden social.

Recorriendo este camino, que ya había sido transitado por los movimientos gays y lésbicos en los años setenta cuando consiguieron que la homosexualidad dejase de ser considerada una enfermedad mental, el texto nos incita a pensar sobre la encrucijada ante la que se encuentra el movimiento transexual: cómo reivindicar su situación de personas sanas sin renunciar a la de que los servicios públicos de salud asuman los costes del tratamiento transexualizador. Responsabilizar a la sociedad del sufrimiento transexual y, por tanto, exigir a sus instituciones que asuman los tratamientos pertinentes tal y como hace el movimiento es, probablemente, el fruto de una situación de transición hacia una toma de conciencia política feminista y sexual más radical, de un lado y, de otro, de una empatía hacia el padecimiento de una parte de las personas transexuales. Pero el sufrimiento, ya sea de los transexuales, de las mujeres, o de otros colectivos oprimidos, solo irá desapareciendo en la medida en que se produzcan cambios estructurales en la sociedad.

Bebiendo de esa preocupación política y de ese activismo académico, el artículo de Paola Sesia, "Derechos humanos, salud y muerte materna: características, potencial y retos de un nuevo enfoque para lograr la maternidad segura en México", desvela también cómo la desigualdad social penetra y articula la práctica biomédica, apuntando esta vez hacia la responsabilidad que tiene el sistema biomédico institucional en la muerte materna en México, que no casualmente afecta fundamentalmente a las mujeres pobres e indígenas. Tras discutir el concepto mismo de derechos humanos, la problemática derivada de su definición, implementación e instrumentalización, analiza el contexto teórico, político e institucional que ha posibilitado el reconocimiento de la muerte materna como una violación de los derechos humanos de las mujeres durante la maternidad. Haber logrado dicho reconocimiento no es una cuestión baladí ya que afirmar que la muerte materna implica una violación a los derechos humanos significa algo tan importante como convertir la muerte materna en una cuestión de estado y posibilita exigir a este último su responsabilidad en la promoción, garantía y defensa de la salud materna en sus políticas públicas.

Analizar la muerte materna como una violación de los derechos humanos nos introduce en un panorama sumamente complejo, ya que dicho análisis implica al Estado y sus políticas públicas; a la garantía que hace ese Estado de los derechos sexuales y reproductivos de las mujeres, entre otros, el derecho al aborto y al acceso a métodos anticonceptivos; implica a las propias instituciones de salud, que no ejercen una atención médica respetuosa, equitativa y de calidad; e implica, por último, a una sociedad sexista, que violenta las vidas sexuales y reproductivas de las mujeres en el ámbito de la pareja, de la familia y de 
la comunidad. El enfoque es, sin duda, sumamente novedoso y su potencialidad reside en que, más allá del individualismo epistemológico biomédico, más allá de pensar la muerte materna como un hecho aislado sobre el que incidir, nos incita a pensar sobre la desigualdad social y, más en concreto, de sexo-género; sobre una práctica biomédica sexista y clasista; sobre unos servicios de salud deficientes; sobre unas políticas públicas ineficientes. Aplicadas en este caso al Estado mexicano, pero representativas sin duda de una realidad mucho más amplia.

Encuadrado también en el ámbito de los Derechos Sexuales y Reproductivos, aunque abordando otro tipo de problemática, el artículo de Gemma Orobitg, Joan Bestard y Carles Salazar, "El cuerpo (re)productivo: interés económico y altruismo social en las experiencias de un grupo de mujeres donantes de óvulos", nos traslada al campo de las nuevas tecnologías reproductivas y los dilemas y paradojas asociadas a las mismas. Trabajando con las narraciones de un grupo de mujeres donantes de óvulos en una clínica privada de infertilidad en Barcelona, el texto analiza el proceso de resignificación que éstas otorgan al acto de donar, permitiéndonos reflexionar sobre la relación entre tecnología médica y lógica social ya que, de nuevo, lo que se presenta como un asunto estrictamente tecnológico es resuelto siempre en un marco social. En concreto, los autores analizan la importancia de la compensación económica para las donantes y su utilidad a la hora de pensar y de resolver las contradicciones inherentes a la donación de una sustancia biogenética que socialmente construye y justifica una relación de filiación. Al introducir la perspectiva de los sujetos sociales y de su capacidad de agencia, el artículo aporta nuevos elementos que complejizan el debate ético existente en torno a la reproducción asistida y, más en concreto, en torno a la compensación económica que reciben las donantes. Dicha compensación, que contradice aparentemente el altruismo que se supone debe acompañar a la donación, y que hace explícita la mercantilización del cuerpo y alerta sobre sus peligros, se revela sin embargo importante y funcional a la hora de construir el sentido individual de la donación.

La forma como las donantes de óvulos construyen el sentido del acto de donar una parte de su cuerpo nos remite a la contradicción existente en una sociedad que no ha establecido con claridad la primacía del vínculo social sobre el biológico en el establecimiento de los lazos de parentesco y que se mueve arbitrariamente entre ambos. $Y$ es en este marco contradictorio y paradójico en el que ellas elaboran sus experiencias, en un contexto clínico, no podemos olvidar, fuertemente medicalizado, que prima únicamente los aspectos biológicos de la procreación y que además, y el dato no es baladí, está operando sobre el cuerpo de las mujeres, un cuerpo, como ya señalaron los análisis feministas, socialmente apropiado y naturalizado y que, como revela el texto, tiene una dimensión productiva fundamental.

Llegados a este punto del relato, creo haber ilustrado cómo los artículos incluidos en este monográfico inciden y ejemplifican lo que entiendo son algunas de las principales 
aportaciones y potencialidades de la Antropología de la Salud. A estos cuatro artículos les siguen otros dos textos. El primero de ellos recoge la voz de esos "otros" excluidos, silenciados, cuyas aportaciones incitan a atender a esa "ecología de saberes" (De Sousa Santos, 2010) que reivindican los estudios poscoloniales. José Yánez con la colaboración de Andrea Yánez, en "El Sistema Andino de Salud (SAS) en el Estado plurinacional del Ecuador", nos acerca la problemática de los pueblos indígenas, en un texto en el que sujeto político, sujeto identitario y sujeto investigador se funden en una sola voz y nos remite a una situación en la que la reivindicación de lo que el autor denomina un "Sistema Andino de Salud" y el gobierno ecuatoriano "Medicina Tradicional" se convierte en la reivindicación de un derecho colectivo cuyo reconocimiento es necesario de cara a la construcción de un sistema de salud intercultural. José Yánez nos trae la voz del pueblo Kayambi, centrado en la biografía de una de sus parteras, Rosa Cabrera. Los fragmentos de la narración de Mama Rosa que recoge el texto son una auténtica delicia para un análisis antropológico. En ellos se articulan sincretismos, interacciones... $Y$ es que no podría ser de otra manera, a pesar de la reificación con la que el autor nos presenta lo que denomina Sistema Andino de Salud (SAS). Esta reificación hay que entenderla en el contexto político en el que se inscribe el texto. Desde la perspectiva de las nacionalidades y pueblos indígenas, construir teóricamente un sistema médico sólido y unificado probablemente sea necesario para dialogar y negociar políticamente, en pié de igualdad, con ese "otro" sistema médico oficial con el objetivo de participar realmente en la fundación del estado plurinacional e intercultural reconocido en la Constitución de 2008 por el gobierno de Rafael Correa. Y es que, para los pueblos y nacionalidades políticamente organizados de Ecuador, y no solo para ellos, la interculturalidad pasa previamente por el reconocimiento de la plurinacionalidad.

De esta forma, el texto nos incita a pensar sobre los retos teóricos y políticos de un término cada vez más de moda en círculos institucionales, académicos y profesionales, la interculturalidad, que está generando una cantidad ingente de publicaciones y que, a menudo, se confunde con una teoría y una práctica de gestión de la multiculturalidad. La reivindicación de un sistema de salud intercultural resulta de gran interés porque los movimientos indígenas no reivindican solo una mejor y mayor atención a la salud, su derecho a acceder a los avances científicos y disminuir la brecha de pobreza, que también, sino una atención a la salud diferente, una atención de cuyo diseño fueron excluidos puesto que, históricamente, el triunfo del modelo biomédico supuso, entre otras cosas, una jerarquización étnica según la cual los "otros" pueblos no tenían nada que aportar porque eran ignorantes y supersticiosos y, por tanto, sus sistemas médicos tampoco. Por eso, la situación que recoge José Yánez en su texto nos empuja a plantearnos el hecho de que reivindicar un sistema de atención a la salud intercultural nos coloca ante el reto de un cambio paradigmático. La propuesta de un cambio de paradigma no es descabellada si reconocemos, con Junge (2001), que "la biomedicina por sí sola no es capaz de satisfacer 
todas las necesidades de salud de la población. (...). La salud es parte de la experiencia vital de las personas, y la vida no se restringe a sus aspectos fisiológicos; por ende, la salud no puede reducirse al enfoque biomédico" (Junge, 2001:6). Entre otras cosas, cuando Yánez afirma, en relación a la salud del pueblo Kayambi, que el problema seguirá empeorando, en esta zona y en las demás, mientras no haya una verdadera Reforma Agraria no hace más que recordarnos, en 2013, lo que ya Rudolf Virchow, considerado el padre de la medicina social, afirmó en 1848 y que parece haber caído en saco roto: que "La medicina es una ciencia social y la política no es más que medicina a gran escala". Para finalizar, el texto de Emilio González, "La entrevista clínica como intervención cultural", constituye una narración autobiográfica a partir de su ejercicio profesional como psiquiatra en Galicia. El texto de Emilio González tampoco es un artículo académico al uso. Es un relato en primera persona, pero no al modo narcisista posmoderno. En él, experiencia personal, profesional y política se dan la mano, haciendo explícitos unos vínculos que normalmente opaca el lenguaje científico y constituyendo un valiosísimo testimonio para la reflexión y el análisis antropológico. Su relato ilustra, efectivamente, desde una perspectiva histórica, el proceso de medicalización del agro gallego y la responsabilidad de los profesionales biomédicos en dicho proceso; la conformación de una praxis médica basada en la psiquiatrización biologicista y las subsiguientes políticas de salud mental implementadas por la Xunta de Galicia, así como las consecuencias que todo ello está teniendo en la actualidad; las lógicas de lo que la Antropología ha denominado "saberes populares". Desde su lucidez profesional, nos presenta a unos sujetos sociales, los campesinos y campesinas gallegos, con capacidad de agencia, en un sistema en el que, de todas formas, ocupan una posición claramente subalterna. Reivindica, desde y a partir de su práctica profesional, el saber de los pacientes, sin dejar por ello de explicitar la desigualdad subyacente a la relación clínica. Incide en la importancia de la "comunidad", de la identidad y de las redes sociales en la salud.

Es importante incorporar voces provenientes de otros ámbitos profesionales a este campo de reflexión antropológico. Sus relaciones con el antropólogo Marcial Gondar en lo que Emilio González se apresura a denominar "acción-investigación” y no al contrario muestran un modo de intervención sobre la realidad que nos obliga a reflexionar sobre las limitaciones de la práctica antropológica en este campo (y no sólo en este campo...). Cuando el texto alude a la "dificultad de hacer operativas en la praxis cotidiana" las aportaciones de la Antropología y nos conmina a afrontar la "heterogloxia existente en el concepto de Antropología de la Salud" creo que plantea cuestiones aún de gran pertinencia.

¿Cómo buscar lugares de encuentro entre profesionales sanitarios y de la antropología? ¿Cómo buscar un lenguaje común, que no descalifique sistemáticamente la práctica médica pero que tampoco coloque a la antropológica en una posición subordinada a la primera? ¿Cómo realizar esa crítica antropológica radical -etimológicamente hablando, 
de raíz- pero a la vez con capacidad de aplicación a la clínica y al estado de salud de los seres humanos? Poner en diálogo la cosmovisión médica y la antropológica y recuperar la eficacia, sin duda existente, en cada una de ellas, parecería pertinente y de gran utilidad social. 


\section{REFERENCIAS BIBLIOGRÁFICAS}

Comelles, Josep María (1988) “Salud/enfermedad”. En Aguirre, Angel (Ed) Diccionario Temático de Antropología, Barcelona: Editorial Boixareu Universitaria, pp 551-558.

Comelles, Josep María y Martínez, Angel (1993) Enfermedad, cultura y sociedad, Madrid: Editorial Eudema.

De Sousa Santos, Boaventura (2010) Para descolonizar Occidente. Más allá del pensamiento abismal. Buenos Aires: CLACSO.

Sociedad Española de Reumatología (2000) Estudio sobre prevalencia e impacto de las enfermedades reumáticas en la población española (EPISER), http://www.ser.es/ investigacion/Proyectos/Episer/Episer.php

Junge, Patricia (2001) "Nuevos Paradigmas en la Antropología Médica” Cuarto Congreso Chileno de Antropología, 19 al 23 de noviembre de 2001, Campus Juan Gómez Millas de la Universidad de Chile, http://rehue.csociales.uchile.cl/antropologia/congreso/s1201. $\underline{\text { html. }}$

Kleinman, Arthur (1978) "Concept and a model for the comparation of medical systems as cultural systems", Social Science of Medicine, 12: 85-93.

Kleinman, Arthur (1980) Pacients and healers in the context of culture, California: University of California Press.

Martínez, Angel (1996) “Antropología de la salud: una aproximación genealógica”. En Prat, Joan y Martínez, Angel (Eds) Ensayos de Antropología cultural, Barcelona: Editorial Ariel, pp 369-378.

Menéndez, Eduardo (1978) "El modelo médico y la salud de los trabajadores". En: Basaglia et al (Eds.) La salud de los trabajadores, México: Nueva Imagen.

Menéndez, Eduardo (1994) "La enfermedad y la curación. ¿Qué es la medicina tradicional?", Alteridades, 4 (7): 71-83.

Menéndez, Eduardo (2012) “Antropología Médica. Una genealogía más o menos autobiográfica”, Gazeta de Antropología, 28(3), http://hdl.handle.net/10481/22988.

Ministerio de Sanidad, Política Social e Igualdad (2011) Fibromialgia, http://www.msssi. gob.es/profesionales/prestacionesSanitarias/publicaciones/Fibromialgia.htm.

Scott, Joan (1990) "El género: una categoría útil para el análisis histórico”. En Alemang, James y Nash, Mary (Eds.) Historia y género: las mujeres en la Europa moderna y contemporánea, Valencia: Editorial Alfons el Magnanim, pp 23-56.

Valls-Llobet, Carme (2009) Mujeres, salud y poder, Madrid: Ediciones Cátedra. 